\title{
Two-sided Platform Pricing and Competition under Realistic Pricing Structure
}

\author{
Tongyao Jiang \\ School of Economics and \\ Management. Northeast Electric \\ Power University, Jilin, 132011, China \\ a02243340@usu.edu
}

\author{
Jie Chen \\ Division of Business and \\ Management, Beijing Normal \\ University-Hong Kong Baptist \\ University United International \\ College, Zhuhai, 519085, China
}

\author{
Zhixuan Xiang \\ Chengdu NO.7 High School \\ International Department, \\ Chengdu,610106, China
}

\begin{abstract}
This paper is motivated by the prevailing pricing structure and competition in Chinese food-delivery market. Currently, two oligarchs in China's food-delivery market are Meituan-Dianping Takeout and ELEME Takeout, which accounting for more than $95 \%$ of the market share. Both of these platforms charge a percentage commission based on per-transaction price and involve per-agent fee. So far, few research has been found to make analysis under such realistic pricing structure, and we help to fill this gap by developing a model for platform competition based on the model developed by Armstrong. The results we get under the realistic pricing structure and cost verify the propositions of Armstrong under per-transaction fee, which may stem from that the percentage commission charged on per-transaction price is similar to the per-transaction fee.
\end{abstract}

\section{CCS CONCEPTS}

- Applied computing; • Electronic commerce; • E-commerce infrastructure;

\section{KEYWORDS}

Two-sided platforms, network effects, pricing structure, duopoly competition

\section{ACM Reference Format:}

Tongyao Jiang, Jie Chen, and Zhixuan Xiang. 2021. Two-sided Platform Pricing and Competition under Realistic Pricing Structure. In 2021 th International Conference on E-Business and Internet (ICEBI 2021), October 15-17, 2021, Singapore, Singapore. ACM, New York, NY, USA, 5 pages. https://doi.org/10.1145/3497701.3497733

\section{INTRODUCTION}

The intermediaries or "platforms" provide a place for two groups of agents in many markets interact with each other, and platforms try to get these two sides on board by charging properly each side. Throughout this dissertation, the term two-sided platform will be used to refer to the situation when two groups of participants need to trade through the platform, and the benefits of one group of

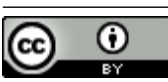

This work is licensed under a Creative Commons Attribution International 4.0 License.

ICEBI 2021, October 15-17, 2021, Singapore, Singapore

(C) 2021 Copyright held by the owner/author(s)

ACM ISBN 978-1-4503-8565-7/21/10.

https://doi.org/10.1145/3497701.3497733 participants joining the platform depend on the number of participants joining the other group of participants on the platform [1]. In this situation, the two groups of agents presenting on one platform can provide each other with network benefits. Moreover, when agents determine to join one, not multiple platforms, this situation is referred to what we called "single-homing".

The central thesis of this paper involves two-sided platform pricing and competition, and what should be emphasized is that our study is under realistic pricing structure. Our analysis is motivated by the prevailing pricing structure and competition in the fooddelivery market. Factors that matter in our analysis are relative size of indirect network externalities, pricing structure, and the homing of agents.

Currently, two oligarchs in China's food-delivery market are Meituan-Dianping Takeout and ELEME Takeout, which accounting for more than $95 \%$ of the market share, and this market is almost a duopoly. Both two platforms compete for attracting buyers and sellers mainly via price, which constitute a duopoly competition. Moreover, a fixed pricing structure has already been formed: both platforms charge a percentage commission based on transaction price. As for the cost, both platforms involve a per-agent cost when agents entering the platforms.

The analyzing approach in this paper is based on a basic model taking the realistic pricing structure of food-delivery platforms into consideration, and we make analysis under the situation of a monopoly platform and duopoly platform with two sides singlehoming. Actually, there are four participants related to the platform: the two sides (the buyers and the sellers),the platform, and the takeaway riders. While our analysis does not take account of takeaway riders, which is unrelated to our analysis. It must be noticed that the major source of platform profit is the commission earnings. However, we will not consider the advertisement income and the logistics income.

By now, little research has been found to survey the realistic pricing structure of food-delivery platform which charges a percentage commission of per-transaction price. Because the pricing structure matters under platform competition, in this paper we build models under this realistic setting, and by doing this study, we attempt to make some contribution to this field of research. In this paper, we explore the impact of such realistic pricing structure on profit-maximizing equilibrium, social welfare, and platform subsidy.

The overall structure of the study takes the form of 4 chapters, and the paper begins by this introductory chapter. It will then go on to lay out a number of longitudinal studies involving two-sided 
platforms. The third chapter is concerned with the methodology used for this study. Finally, the conclusion gives a summary and some suggestions about this study.

\section{LITERATURE REVIEW}

While most of the literature researches price strategy and price competition between two side platforms, the largest difference between these papers and ours is that our analysis is under the realistic pricing structure. Our paper mainly follows the model of Armstrong [1] but we modify the model by adding some variables and by changing some assumptions.

The study of two-sided platform can be traced to some ealier but still important works by Caillaud and Jullien [2], Rochet and Tirole [3], and Armstrong [1], a great number of studies has been done on two-sided platforms. Rochet and Tirole [3] build a model of platform competition on two-sided markets. They assume that the platform charged fixed fees and per-transaction fees when consumers are single-homing. However, in today's real-life market, most platform don't charge the sellers for joining the platform. This situation can be seen from our example of food delivery platforms.

Caillaud and Jullien [2] consider price competition between two platforms providing intermediation services, and they focus on the multi-homing issue. However, what it studies is the situation happens at an earlier stage of the platform, and it analyzes this issue under the assumption of homogeneous agents. What we want to explore is the established platforms whose pricing structures are fixed, and we assume heterogenous agents.

Gabszewicz and Wauthy [4] model duopoly competition between two platforms in a two-sided market where both sides of the agents are heterogeneous similar to us and are allowed to multihoming. However, this paper does not use flexible pricing. Our real-life examples (i.e., Chinese food delivery platforms) will use a percentage commission fee charging on price per to further the study of these questions.

Armstrong [1] discusses three settings: the first one is under a monopoly platform, the second one is on competing platforms when two sides single homing, and the third model is about "competitive bottlenecks" where one side multi-home. The determinants of equilibrium prices for each model are explored in this paper. This paper finds that if consumers on one side can be multihoming, platforms will make the single-homing side more monopoly pressure and compete on it. Although these cases analysis the twosided markets, it does not consider the case of multihoming, and It doesn't fit the actual market. In our model, we follow this paper to build a model for welfare and profit on the realistic pricing structure. We first assume the platform is a monopoly. Armstrong [1] finds that profit-maximizing outcomes are related to the demand elasticity of participants presenting on the platforms and the network externalities they get. In our food delivery model, we find the social welfare-maximizing commission percentage is equal to the per-agent cost adjusted by the network effect and we modulate downward by a coefficient relating to the participants' demand elasticity. Under the situation of duopoly platforms with two side single-homing, the results we get are very similar to those of Armstrong [1], which is not surprised for the percentage commission charged on per-transaction price is similar to the per-transaction fee.

Choi [5] fills the gap and investigates the competition for market and social welfare that is in two-sided markets when agents can be multi-homing. This paper focuses on tying and finds that tying increases the welfare if there is multi-homing. This analysis can have important implications for recent antitrust cases in industries where multi-homing is prevalent. However, this paper differs from ours in it mainly focuses on the issue of tying and antitrust issues, but not pricing structure.

Choi, Jullien, and LeFouili [6] further the study of tying, and they analyze the benefits and welfare optimization of tying. This paper model competition of the two platforms providing content to spatially differentiated consumers. The certain set of their analysis and the equilibria are related to the situation of both sides multihome. However, the focus of their study is on tying instead of pricing, which differs from us.

Choi and Jeon [7] discuss a leverage theory of tying which is in two-sided markets. Using three cases with different assumptions, this paper identifies requirements that there are some benefits of tying in two-sided markets and explores the meaning of welfare. These findings may provide useful information for the antitrust investigations making policy, through which they could measure the potential efficiencies and harm.

Belleflamme and Peitz [8] review important discoveries from the literature on network effects and two-sided platforms. It lists the basic models and some approaches taken in recent studies are also described. While this paper ignores works on competition policy issues such as the analysis of price-parity clauses, it provides us a general review of the issue of two-sided platforms.

Belleflamme and Peitz [9] follow the model of Armstrong [1]. This paper discuss the price and surplus influence of multihoming, and further compares the case when one side single while the other sides multihome to the situation when both sides of the market singlehoming. They find there are the same possibility of two results, including benefiting but not hurting the multi-homing side. They identify it is important to research platform competition when both sides multi-home. This paper assumes platforms charge access fees to each side. We want to explore these issues when the platforms charge a percentage commission per transaction.

Bakos and Halaburda [10] help to fill gap where both side of the market have the possibility to multihome. It uses the Hoteling model to study the competition of platforms with two sides as other literature but focuses on the case of multihoming. It develops a model for platform competition between two-sided platforms when both sides multi-home. They find the degree of interdependence is largely determined by the effectiveness of the second meeting. However, this paper differs from ours in that it focuses on the effectiveness of subsidies other than platform competition and pricing.

\section{MODEL}

In the model part, first I'll introduce our basic model; then I'll make analysis under the situation of a monopoly platform and duopoly competition with two sides single-homing. 
Table 1: Variables and explanations:

\begin{tabular}{ll}
\hline Variable & Explanation \\
\hline $\mathrm{U}$ & utility \\
$\beta$ & percentage of commission \\
$\mathrm{b}$ & per-transaction price \\
$\alpha$ & sensitivity to network effect \\
$\mathrm{n}$ & number of participants joining the platform from the other side of the market \\
\hline
\end{tabular}

\subsection{The basic model}

In this section, I'll give a basic model of two-sided platform with the realistic pricing structure. This model is based on the model built by Armstrong [1], and I make some modifications on the pricing satructure and platform costs. The analysis in this part serves as a benchmark for the forthcoming analysis of other settings. In this model, there are two types of agents, indexed by $j=1$ and 2 . These two types of agents interact with each other when they present on the same platform. In the real-life example of food-delivery market, the two types of agents can be customers who order the take-out and firms that provides take-out services. Currently, the two major food-delivery platforms in China are Meituan-Dianping Takeout and ELEME Takeout. In this model, considering the real-life setting, there are two intermediaries, platform $i$ and $j$, indexed by $i=i$ and $j$. The two platforms compete for obtaining a larger market share via price.

In our model, the net utility is defined as: $u_{j}^{i}=\left(1-\beta_{j}^{i}\right) b_{j}^{i} \alpha_{j}^{i} \times n^{i}$. $u_{j}^{i}$ refers to the utility of agent $\mathrm{j}$ on platform $\mathrm{i}$, where $j=1,2 ; i=$ $i, j$. Different from Armstrong [1], the platforms charge a percentage commission based on per-transaction price, and we introduce a new parameter, $\beta$, which refers to the percentage of commission charged by the platforms. Therefore, $\left(1-\beta_{j}^{i}\right)$ is the percentage left to the agents. Another new variable we introduced is b, which is the pertransaction price, and it conforms to the uniform distribution from 0 to 1 . Therefore, $(1-\beta) \mathrm{b}$ is how much platform charge from per transaction. Another difference between this model and Armstrong [1] is that the platforms in our model charge a per-agent fee when the agent joins the platform. Similar to Armstrong [1], we use $\alpha$ to measure the sensitivity to network effect, and $n$ is the number of participants joining the platform from the other side of the market.

Table 1 shows the key variables and modeling setup:

\subsection{Monopoly platform}

First, we will start our analysis from a market where there is only a single platform. Though this setting is not commonly applied to most of the real-life instances of what we called two-sided platforms, this relatively simple setting can serve as a good beginning for more complicated setting in this paper.

First, let's formulate the net utility. Assume there are two groups of agents, denoting group 1 and group 2. If there are $\mathrm{n} 1$ and $\mathrm{n} 2$ of group 1 and 2 participants joining the platform,following the basic model presented before, the net utility in the monopoly platform for these two groups of participants can be expressed as

$$
\begin{aligned}
& u_{1}=\left(1-\beta_{1}\right) b_{1} \alpha_{1} \times n_{2} \\
& u_{2}=\left(1-\beta_{2}\right) b_{2} \alpha_{2} \times n_{1}
\end{aligned}
$$

These two expressions describe utility is determined by a function of the number of agents on the platform. For further analysis, we define $\mathrm{n}$ as a function of $\mathrm{u}$ :

We assume that $n_{1}=\emptyset_{1}\left(u_{1}\right), n_{2}=\emptyset_{2}\left(u_{2}\right)$, and $\emptyset_{1}, \emptyset_{2}$ are increasing functions.

Solve for $\beta_{1}=1-\frac{u_{1}}{b_{1} \alpha_{1} n_{2}}, \beta_{2}=1-\frac{u_{2}}{b_{2} \alpha_{2} n_{1}}$ We assume platform 1 and platform 2 respectively charge per-agent cost of f1 and f2, therefore the total cost equals to $f_{1} n_{1}+f_{2} n_{2}$.

Platform profit equals to the number of agents multiply commission minus cost of the two platforms, which can be formulated as $\left(\beta_{1} b_{1}-f_{1}\right) n_{1}+\left(\beta_{2} b_{2}-f_{2}\right) n_{2}$.

If we consider the payoffs in terms of utilities instead of prices, then the implicit price for the first group of participants can be expressed in the following expression: $\pi\left(u_{1}, u_{2}\right)=$ $\emptyset_{1}\left(u_{1}\right)\left[\left(1-\frac{u_{1}}{b_{1} \alpha_{1} n_{2}}\right) b_{1}-f_{1}\right]+\emptyset_{2}\left(u_{2}\right)\left[\left(1-\frac{u_{2}}{b_{2} \alpha_{2} n_{1}}\right) b_{2}-f_{2}\right]$ Then let the sum of consumer surplus for these two groups of agents be $v_{i}\left(u_{i}\right)$, where vi conforms to the envelope theorem, namely, $v_{i}\left(u_{i}\right)$ identically equals to $\emptyset_{i}\left(u_{i}\right)$.

Welfare equals to profit plus consumer surplus: $w=\pi\left(u_{1}, u_{2}\right)+$ $v_{1}\left(u_{1}\right)+v_{2}\left(u_{2}\right)$ The utilities when the welfare of these two groups is maximized satisfy the following expressions: $u_{1}=$ $\alpha_{1} n_{2}\left(b_{1}-f_{1}+1-\frac{1}{\alpha_{1} n_{2}}\right) ; u_{2}=\alpha_{2} n_{1}\left(b_{2}-f_{2}+1-\frac{1}{\alpha_{2} n_{1}}\right)$;

From (1)(2), commission percentage $\beta$ meets the following expressions: $\beta_{1}=\frac{1}{b_{1}}\left[f_{1}+\left(\frac{1}{\alpha_{1} n_{2}}-1\right)-\frac{\emptyset_{1}\left(u_{1}\right)}{\emptyset_{1}\left(u_{1}\right)}\right] ; \beta_{2}=\frac{1}{b_{2}}\left[f_{1}+\right.$ $\left.\left(\frac{1}{\alpha_{2} n_{1}}-1\right)-\frac{\emptyset_{2}\left(u_{2}\right)}{\emptyset_{2}{ }^{\prime}\left(u_{2}\right)}\right]$ Therefore, the social welfare-maximizing commission percentage is equal to the per-agent cost $\left(f_{1}, f_{2}\right)$ adjusted by the network effect and adjusted downward by a factor relating to the elasticity of the number of agents participating on the platform. We can also get the same expressions using the Lerner indexes and elasticities:

Proposition1:

$$
\begin{gathered}
\eta_{1}=\frac{u_{1}^{\prime} \phi_{1}\left(u_{1}\right)}{\emptyset_{1}{ }^{\prime}\left(u_{1}\right)}=\frac{u_{1}^{\prime} \phi_{1}\left[\left(1-\beta_{1}\right) b_{1} \alpha_{1} n_{2}\right]}{\phi_{1}^{\prime}\left[\left(1-\beta_{1}\right) b_{1} \alpha_{1} n_{2}\right]} ; \\
\eta_{2}=\frac{u_{2}^{\prime} \phi_{2}\left(u_{2}\right)}{\emptyset_{2}{ }^{\prime}\left(u_{2}\right)}=\frac{u_{2}^{\prime} \phi_{2}\left[\left(1-\beta_{2}\right) b_{2} \alpha_{2} n_{1}\right]}{\phi_{2}{ }^{\prime}\left[\left(1-\beta_{2}\right) b_{2} \alpha_{2} n_{1}\right]}
\end{gathered}
$$

The profit-maximizing pair of utilities satisfy the following expressions:

$$
\begin{aligned}
& \frac{u_{1}-\frac{1}{b_{1}}\left(1-f_{1}-\frac{1}{\alpha_{1} n_{2}}\right)}{u_{1}}=\frac{1}{\eta_{1}} \\
& \frac{u_{2}-\frac{1}{b_{2}}\left(1-f_{2}-\frac{1}{\alpha_{2} n_{1}}\right)}{u_{2}}=\frac{1}{\eta_{2}}
\end{aligned}
$$

Looking at these expressions, we find that under profitmaximizing results, platforms can offer group 1 a subsidy, if group 
1 has a high elasticity of demand and the internet externality is large.

\subsection{Duopoly platform when two sides single-homing}

In this part, we'll extend the monopoly platform model to duopoly platforms when two sides single-homing. Similarly, we also assume that there are two groups of agents: group1 and group 2. Using Hoteling model, we assume the two platforms are respectively at the endpoints of an interval with a unit length, and that each side of mass 1 , therefore the total number of group 1 on two platforms is 1 , and the same applies to the group 2. Therefore, we have $n_{1}^{i}+n_{1}^{j}=1$, $n_{2}^{i}+n_{2}^{j}=1$.

The utilities on platform i can be expressed as following:

$$
\begin{aligned}
& u_{1}^{i}=\left(1-\beta_{1}\right) b_{1} \alpha_{1} n_{2}^{i}-t_{1} n_{1}^{i} \\
& u_{2}^{i}=\left(1-\beta_{2}\right) b_{2} \alpha_{2} n_{1}^{i}-t_{2} n_{2}^{i}
\end{aligned}
$$

The same applies to platform $\mathrm{j}$. Using the Hoteling model, we can get the two expressions bellow, where $t_{1}, t_{2}$ are the transportation cost and $\mathrm{t} 1, \mathrm{t} 2$ are more than zero.

$$
\begin{aligned}
& n_{1}^{i}=\frac{1}{2}+\frac{u_{1}^{i}+u_{1}^{j}}{2 t_{1}} \\
& n_{2}^{i}=\frac{1}{2}+\frac{u_{2}^{i}+u_{2}^{j}}{2 t_{2}}
\end{aligned}
$$

Putting together expression (5)(6) and expression (7)(8), and using the fact that $n_{1}^{i}+n_{1}^{j}=1$ and $n_{2}^{i}+n_{2}^{j}=1$, then we will get the two implicit expressions for market shares:

$n_{1}^{i}=\frac{1}{2}+\frac{\left(1-\beta_{1}\right) b_{1} \alpha_{1} \times\left(n_{2}^{i}-n_{2}^{j}\right)}{2 t_{1}}=\frac{1}{2}+\frac{\left(1-\beta_{1}\right) b_{1} \alpha_{1} \times\left(2 n_{2}^{i}-1\right)}{2 t_{1}}$

$n_{2}^{i}=\frac{1}{2}+\frac{\left(1-\beta_{2}\right) b_{2} \alpha_{2} \times\left(n_{1}^{i}-n_{1}^{j}\right)}{2 t_{2}}=\frac{1}{2}+\frac{\left(1-\beta_{2}\right) b_{2} \alpha_{2} \times\left(2 n_{1}^{i}-1\right)}{2 t_{2}}$

Fixing the commission percentage of group 2, (9)(10) indicate that one more group 1 participate presenting on a platform attracts $\frac{\left(1-\beta_{2}\right) b_{2} \alpha_{2}}{t_{2}}$ additional group 2 participates to join the platform.

$$
\begin{aligned}
& \frac{\partial n_{1}^{i}}{\partial n_{2}^{i}}=\frac{\left(1-\beta_{1}\right) b_{1} \alpha_{1}}{t_{1}} \\
& \frac{\partial n_{2}^{i}}{\partial n_{1}^{i}}=\frac{\left(1-\beta_{2}\right) b_{2} \alpha_{2}}{t_{2}}
\end{aligned}
$$

The strength of the indirect network effects is measured by ratio: $\frac{\left(1-\beta_{1}\right) b_{1} \alpha_{1}}{t_{1}}$, and it determines the number of active platforms. Because when network effect is sufficiently larger than transportation cost, platform compete in prices and only one of the platforms remains active.

To focus on market-sharing equilibria, we assume small network effect compared with transportation cost. To ensure the secondorder conditions are satisfied, we impose the strong limitation that

$$
4 \mathrm{t} 1 \mathrm{t} 2>\left(1-\beta_{1}\right) \cdot\left(1-\beta_{2}\right) \mathrm{b} 1 \alpha_{1} \cdot \mathrm{b} 2 \alpha_{2}
$$

which is not only necessary but also sufficient for an equilibrium under this setting to exist.
Platform profit can be expressed as the following expression: $\pi=$ $\left(\beta_{1}^{i} b_{1}-f_{1}\right) n_{1}^{i}+\left(\beta_{2}^{i} b_{2}-f_{2}\right) n_{2}^{i}=\left(\beta_{1}^{i} b_{1}-f_{1}\right)\left[\frac{1}{2}+\frac{\left(1-\beta_{1}\right) b_{1} \alpha_{1} \times\left(2 n_{2}^{i}-1\right)}{2 t_{1}}\right]+$ $\left(\beta_{2}^{i} b_{2}-f_{2}\right)\left[\frac{1}{2}+\frac{\left(1-\beta_{2}\right) b_{2} \alpha_{2} \times\left(2 n_{1}^{i}-1\right)}{2 t_{2}}\right]$ First-order conditions of profit maximization in symmetric equilibrium can be expressed in the following implicit expressions:

$$
\begin{aligned}
& \frac{1}{2} b_{1}+\frac{b_{1} \alpha_{1} b_{2} \alpha_{2}}{2 t_{1} t_{2}}\left\{\left(1-\beta_{2}^{i}\right)\left[\left(2 n_{1}^{i}-1\right) b_{1}-\left(\beta_{1}^{i}-f_{1}\right)\right]\right. \\
& \left.+\left(\beta_{2}^{i} b_{2}-f_{2}\right)\left(2 n_{2}^{i}-1\right)\right\}=0 \\
& \frac{1}{2} b_{2}+\frac{b_{1} \alpha_{1} b_{2} \alpha_{2}}{2 t_{1} t_{2}}\left\{\left(1-\beta_{1}^{i}\right)\left[\left(2 n_{2}^{i}-1\right) b_{2}-\left(\beta_{2}^{i}-f_{2}\right)\right]\right. \\
& \left.+\left(\beta_{1}^{i} b_{1}-f_{1}\right)\left(2 n_{1}^{i}-1\right)\right\}=0
\end{aligned}
$$

Proposition 2: Obviously, $\beta 1$ and $\beta 2$ can be solved. Suppose expression (13) holds, the model under two-sided single-homing has a unique and symmetric equilibrium. Similarly, the social welfaremaximizing commission percentage is related to the per-agent cost $\left(f_{1}, f_{2}\right)$ adjusted downward by the network effect and the elasticity of the group's participation.

Compared with the monopoly, we observe that competition makes platforms emphasize more on the internet externalities, for the lost agent of one platform will join the competitor's platform. When platforms are symmetric, in equilibrium, each platform has half of the market share and obtain half of the total profit.

In the above situation, the results we have get are very similar to those of Armstrong [1], which is not surprising because the pricing structure in our model, the percentage commission charged on per-transaction price, is similar to the per-transaction fee. However, our assumptions are closer to the real-life setting, and our analysis proves that the propositions apply to real-life market.

\section{CONCLUSION}

Throughout this paper, we analyze the pricing and competition of platform with two sides under realistic pricing structure, and we take into consideration the indirect network effect. Following Armstrong [1], we build a model taking into consideration of the realistic setting of Chinese food-delivery platform. In our model, platforms charge a percentage commission on per-transaction price and incur a per-agent fee when agents join the platforms, which are the most important difference between our model and Armstrong [1]. In this paper, we mainly analyze two situations: one is monopoly platform, which serves as a benchmark, and the other is duopoly platform competition with both sides single-homing.

Our analysis is motivated by the prevailing pricing structure of Chinese food-delivery platforms. We modify the model in Armstrong [1] by using realistic pricing structure which platforms charge a percentage commission on a per-transaction price. We find that a monopoly platform can offer the group with a high elasticity of demand with subsidies under the profit-maximizing situation. We also find that the model under two-sided single-homing has a unique and symmetric equilibrium, and the social welfaremaximizing commission percentage is related to the per-agent cost $\left(f_{1}, f_{2}\right)$ adjusted downward by the network effect and the elasticity of the group's participation. The results we get under the realistic 
pricing structure and cost are very similar to those under a pertransaction fee. However, our analysis contributes to the analysis of realistic setting of Chinese food-delivery platform.

There are at least two limitations in our current model. First, we carry out our analysis under the assumption of symmetric platforms. However, in the real market, the platforms are usually asymmetric with slightly different pricing structure and cost. Additionally, in this paper, we do not analyze the situation of duopoly platform competition when two sides are allowed multihoming under the realistic pricing structure because of the length of this paper. Future studies could further explore these issues with asymmetric platforms and under the situation of two-sides multihoming.

\section{REFERENCES}

[1] Armstrong, M. (2006). Competition in Two-Sided Markets. The RAND Journal of Economics, 37(3), 668-691.
[2] Caillaud, B., \& Jullien, B. (2003). Chicken and egg: competition among intermediation service providers. The RAND Journal of Economics, 34(2), 309-328.

[3] Rochet, J. C., \& Tirole, J. M. P. (2003). PLATFORM COMPETITION IN TWO-SIDED MARKETS. Journal of the European Economic Association, 1(4), 990-1029.

[4] Gabszewicz, J., \& Wauthy, X. (2004). Two-sided markets and price competition with multi-homing. Research Papers in Economics.

[5] Choi, J. P. (2010). Tying in two-sided markets with multi-homing. Journal of Industrial Economics, 58(3), 607-626.

[6] Choi, J. P., Jullien, B., \& Lefouili, Y. (2017). Tying in Two-Sided Markets with Multi-Homing: Corrigendum and Comment. Journal of Industrial Economics, 65(4), 872-886.

[7] Choi, J. P., \& Jeon, D.-S. (2021). A Leverage Theory of Tying in Two-Sided Markets with Nonnegative Price Constraints. American Economic Journal: Microeconomics, 13(1), 283-337.

[8] Belleflamme, P., \& Peitz, M. (2018). Platforms and network effects. Chapters, 286-317.

[9] Belleflamme, P., \& Peitz, M. (2019). Platform competition: Who benefits from multihoming? International Journal of Industrial Organization, 64, 1-26.

[10] Bakos, Y., \& Halaburda, H. (2020). Platform Competition with Multihoming on Both Sides: Subsidize or Not? Management Science, 66(12), 5599-5607. 